\title{
On-line Detection of Rotor Eccentricity and Demagnetization Faults in PMSMs based on Hall- effect Field Sensor Measurements
}

\author{
Yonghyun Park*, Daniel Fernandez ${ }^{* *}$, Sang Bin Lee*, Doosoo Hyun*, Myung Jeong*, Suneel Kumar Kommuri*, \\ Changhee Cho ${ }^{* * *}$, David Reigosa**, and Fernando Briz ${ }^{* *}$ \\ *Department of Electrical Engineering, \\ Korea University, Seoul, Korea \\ Email: sangbinlee@korea.ac.kr \\ ${ }^{* *}$ Department of Precision Mechanical \\ Engineering, Gyeonggi College of \\ Science \& Technology, Siheung, Korea
}

\begin{abstract}
Rotor eccentricity and local demagnetization in permanent magnet synchronous motors (PMSMs) increases unbalanced magnetic pull and motor vibration resulting in accelerated aging of motor components. If the asymmetry in the rotor remains undetected, it can increase in severity, and increase the risk of stator-rotor contact, which causes forced outage of the motor and driven process. Detection of PMSM rotor asymmetry currently relies on off-line testing and on-line vibration/current spectrum analysis. However, they are inconvenient or cannot provide reliable detection of rotor faults for all PMSM designs. In this paper, the feasibility of using the signals from analog Halleffect field sensors for detecting eccentricity and local demagnetization is investigated. It is shown that Hall sensors present in machines for motion control can be used for directly measuring the variation in the flux inside the motor due to rotor magnetic asymmetry with minimal hardware modifications. 3dimensional (3D) finite element analysis (FEA) and experimental results performed on an interior PMSM (IPMSM) show that the proposed method can provide sensitive and reliable detection of dynamic/mixed eccentricity and local PM demagnetization.
\end{abstract}

Index Terms-Condition Monitoring, Demagnetization, Finite Element Analysis, Hall Effect Field Sensor, Permanent Magnet Synchronous Motor, Rotor Eccentricity.

\section{INTRODUCTION}

Permanent magnet synchronous machines (PMSMs) are replacing induction motors in many applications due to their benefits on efficiency, power density, and ease of control. With the recent increase in the demand for PMSMs, there has been a lot of research activity on detection of PMSM drive system faults such as rotor eccentricity, PM demagnetization, and load alignment/unbalance-related failures [1]-[12]. There are three types of rotor eccentricity: static, dynamic, and mixed eccentricity (SE, DE, and ME). SE is a condition where the position of the minimum radial air gap is fixed. It can be caused by imperfections introduced during manufacturing or assembly, and its level rarely changes over time. DE is a condition where the position of the minimum air gap rotates with the rotor, and is produced by imperfections in the rotor, worn bearings, bent shaft, etc. SE and DE usually co-exist as ME, and its inherent level is within $10 \%$ of the air gap [13]. Irreversible local demagnetization in the PMs can be caused by a combination of thermal stress, demagnetizing magneto-motive force, mechanical stress (chipping or cracking), or disintegration due to corrosion/oxidation [1]-[4], [11]. Eccentricity (DE and ME) and local demagnetization cause unbalanced magnetic pull, which results in increased vibration, acoustic noise, bearing wear, and/or rotor deflection. Asymmetry in the rotor must be detected since they can increase in severity over time, increasing the risk of stator-rotor contact, which causes serious damage to the PM, core, and stator winding insulation [11], [13].

A number of researchers have developed techniques for detecting rotor asymmetry due to eccentricity and local demagnetization to improve the performance and reliability of PMSM drive systems [1]-[12]. The traditional offline tests for detecting eccentricity include the dial indicator and feeler gauge tests, where the out of roundness of the rotor or the airgap are measured [11], [13]. Local PM demagnetization can be easily detected with a gaussmeter scan on the rotor surface with the rotor removed [11]. Although these tests can provide precise assessment of eccentricity and demagnetization, they cannot be applied frequently, as they require full or local motor disassembly, manual rotor rotation, and/or specialized test equipment or setup. In [11], an off-line test for rotor faults based on injecting a signal using the inverter is presented; however, it requires the motor to be at standstill for testing.

Methods for on-line detection of eccentricity and local demagnetization have been studied in [3]-[10] for in-service assessment of rotor asymmetry. Estimation of the magnetic flux based on the mathematical model has been studied for detection of PMSM rotor faults in [3]-[4]; however, they are known to be sensitive to non-ideal modeling errors and variation in motor parameters. In [5]-[6], on-line monitoring of the rotor rotational speed frequency, $f_{r}$, sidebands of the fundamental frequency $\left(f_{s}\right)$, $f_{\text {ecc }}$, given by

$$
\begin{gathered}
f_{r}=k \cdot f_{s} / p, \\
f_{e c c}=f_{s} \pm f_{r}=(1 \pm k / p) f_{s},
\end{gathered}
$$

from the vibration, shaft voltage, or flux spectra have been studied, where $p$ is the number of pole pairs, and $k$ is a positive integer. However, the main limitation is the requirement of physical access to the motor for walk-around type monitoring or permanent installation of sensors. If portable equipment is used for monitoring, the maintenance personnel are exposed to safety risks, especially for cases where the motor is being operated in a hostile environment. Retrofitting sensors and cables to the 
motors for permanent installation also increases the system cost and degrades system reliability. Most of the recent studies, therefore, focus on applying motor current signature analysis (MCSA), where the $f_{\text {ecc }}$ component in the stator current spectrum is monitored. MCSA can provide remote, online monitoring from the inverter using existing sensors, and is independent of the motor mathematical model [3]-[4], [7]-[10]. Although MCSA has many advantages, the detectability of the fault depends on the winding configuration [1], and a complex timefrequency analysis technique is required for motors operating under nonstationary conditions, where the input frequency or load varies [9]-[10]. In addition, non-idealities in the PMSM drive system such as misalignment, load unbalance, or other mechanical asymmetries produce sidebands components identical to $f_{e c c}$, and there is no practical means of separating them [3]-[4].

It is concluded from the previous discussion that there are many limitations to applying existing PMSM eccentricity detection methods in the field. Therefore, development of new test methods able to provide remote, on-line detection of eccentricity and demagnetization faults independent of motor design or model and without additional sensors, is highly desirable. The main idea of this work is to take advantage of the Hall sensors required in the motor motion control. The feasibility of using an analog Hall sensor for detection of rotor asymmetry is evaluated in this paper. A 3D FEA and experimental study on IPMSMs show that the proposed method can provide sensitive and reliable detection of DE, ME and local demagnetization faults with minimal hardware modifications.

\section{ANALYSiS OF HALL SENSOR MEASUREMENTS UNDER ECCENTRICITY AND LOCAL DEMAGNETIZATION CONDITIONS}

\section{A. Hall Sensor Measurement}

Since the absolute position of the rotor is required for PMSM control, many PMSM systems employ an incremental encoder and three digital Hall-effect sensors located 120 electrical degrees apart for obtaining the initial position for motor starting. The signals measured with these sensors are usually digitized, as shown in Fig. 1, for simplifying the hardware and for noise immunity. There are also commercial PMSM drives where the option of using the analog Hall sensors is offered [14]-[15]. The PM leakage flux inside the motor measured with the analog Hall sensors (Fig. 1) contains information that could be used for control or monitoring purposes. The use of analog Hall sensor signals for improving control performance and PM temperature estimation have recently been investigated [17]-[22]. Since analog Hall sensors provide measurements of the flux inside the motor, it is expected that rotor eccentricity and local demagnetization can be detected by monitoring anomalies in the flux pattern. If the PMSM system is equipped with analog Hall sensors, installation of additional sensors or hardware is not required to implement the method proposed in the paper. For cases where the Hall sensor measurements are digitized, replacement of the sensor or modification of the system is required, as will be shown in IV.

A 3 dimensional (3D) FEA was performed on a $7.5 \mathrm{~kW}, 6$ pole IPMSM model shown in Fig. 2 to observe how the flux measurements change with rotor eccentricity and local PM demagnetization. The coordinate system used for the PM flux in the tangential, $x$, radial, $y$, and axial, $z$, directions are shown. The Hall sensor was fixed to the stator and located $4 \mathrm{~mm}$ in the radial $(y)$ direction, and $5 \mathrm{~mm}$ in the axial (z) direction with respect to the geometric center of the PM surface. The variation in the PM flux measurement in the $x, y$, and $z$ directions with the PM temperature was investigated in detail in [22]. The results show that the measurements change with PM flux strength regardless of the direction of flux measurement. The flux in the axial (z) direction was used in this work, as it was shown in [22] that it is relatively sensitive to the PM field while being insensitive to the field produced by the stator endwinding current when installed at the given position. It should be noted that $x$ and $y$ direction flux would lead to similar performance when used for eccentricity detection.

A 3D FE electromagnetic transient analysis was performed with ANSYS Maxwell on the full machine model. The 3D model includes the stator end-winding geometry, as shown in Fig. 2(b), and the nonlinear model of the electromagnetic materials. Flux density of PM leak flux is measured in an environment modeled as an air volume, with a reduced mesh size of $0.5 \mathrm{~mm}$, and with an average mesh size of $0.58 \mathrm{~mm}$ for the full model. The $x-, y$-, and z-axis flux density patterns as a function of rotor flux angle measured with the Hall sensor are shown in Fig. 3(a). SE, DE and ME conditions were produced

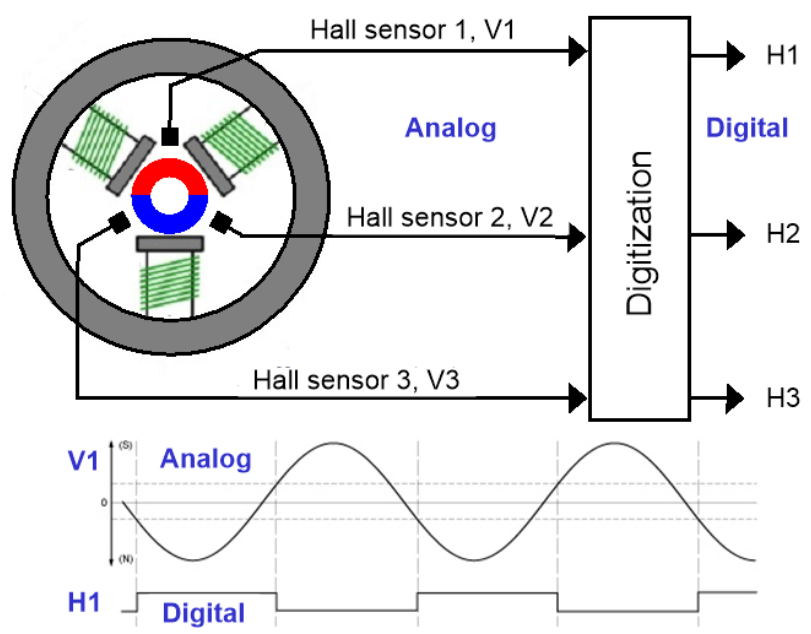

Fig. 1 Schematic of Hall effect field sensor analog measurement and digitization for obtaining rotor position

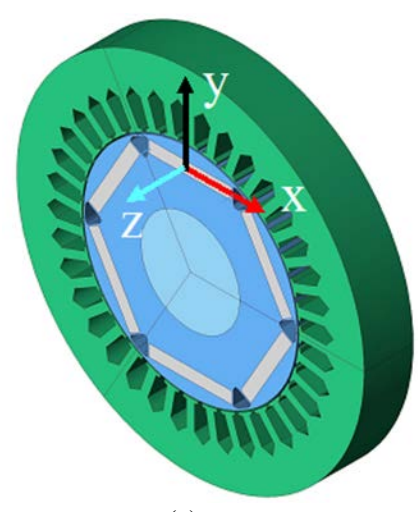

(a)

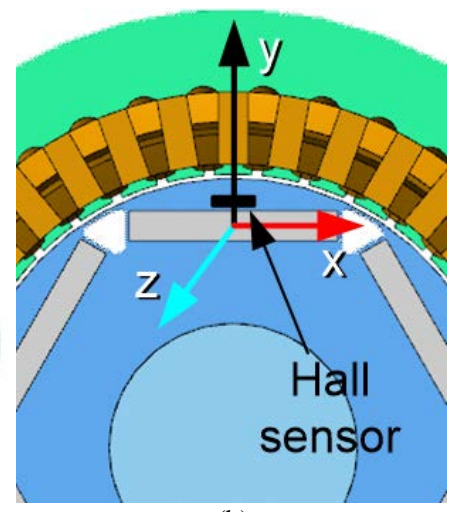

(b)
Fig. 2 3D FE model of $7.5 \mathrm{~kW}, 6$ pole IPMSM with (a) tangential, $x$, radial, $y$, and axial, $z$, coordinate system directions, and (b) Hall sensor location shown 


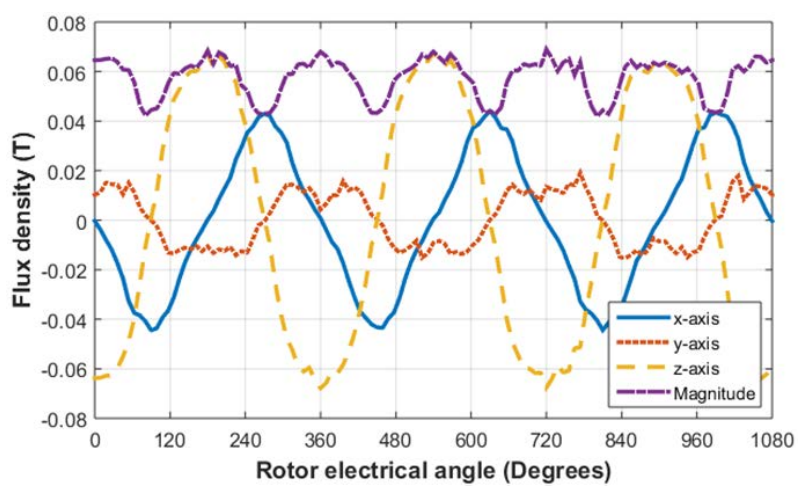

(a)

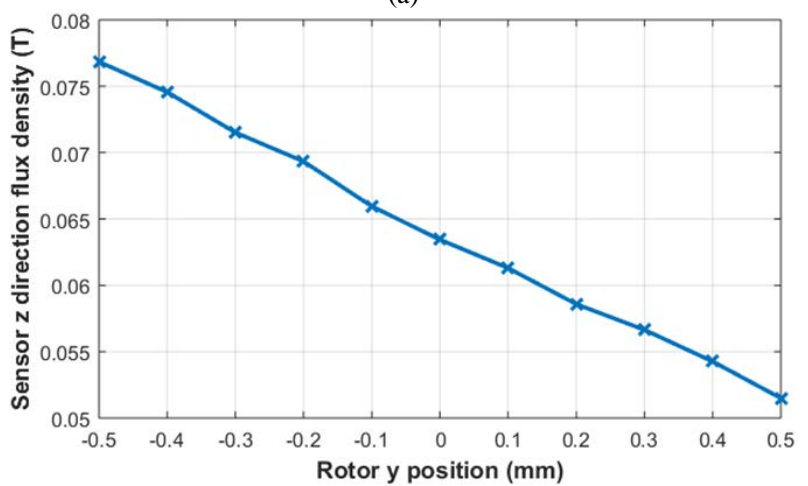

(b)

Fig. 3 (a) $x$-, $y$-, and $z$-axis flux density as a function of rotor electrical angle; (b) axial (z) direction peak flux measurement as a function of rotor radial $(y)$ location (corresponds to $-62.5 \%$ and $+62.5 \%$ rotor eccentricity)

by offsetting the center of the rotor with respect to the center of the stator (SE) and offsetting the center of rotation with respect to the center of the rotor (DE, ME). The motor was operated at $1000 \mathrm{rpm}$ and rated load conditions for both concentric and eccentric conditions.

\section{B. Analysis under Fault Conditions}

For a PMSM with eccentricity, the radial location of the rotor (and PM) changes with rotor position depending on the type and severity of eccentricity. This changes the relative distance between the Hall sensor and PM, since the Hall sensor is fixed to the stator, whereas the eccentric rotor rotates with respect to its own center of rotation. To perform a qualitative analysis on how the Hall sensor measurement changes with eccentricity, the

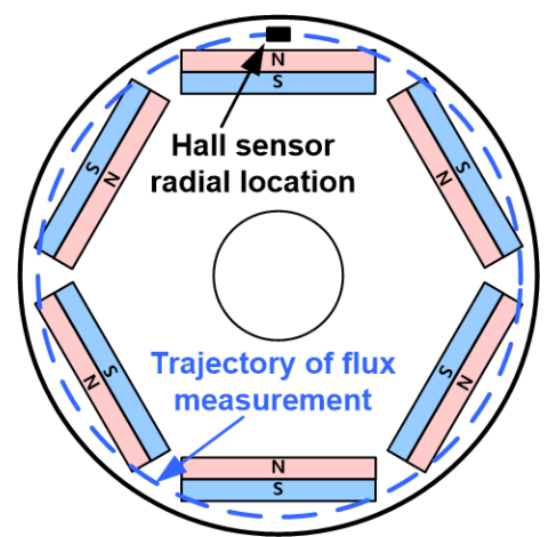

Fig. 4 Hall sensor location, and trajectory of relative radial position between Hall sensor and PMs for concentric rotor

axial (z) direction flux as a function of the rotor (or PM) radial (y) location was obtained from the 3D FEA. The measured analog signal resembles the sinewave signals shown in Figs. 1 and 6 , where the $+/-$ peaks are measured when a PM passes the sensor. The rotor was moved in discrete steps of $0.1 \mathrm{~mm}$ between -0.5 and $+0.5 \mathrm{~mm}$, which corresponds to $-62.5 \sim$ $+62.5 \%$ eccentricity for the IPMSM with a $0.8 \mathrm{~mm}$ airgap. The peak amplitude of the $z$ direction flux analog measurements decreases almost linearly with rotor radial displacement, as shown in Fig. 3(b). For this particular IPMSM, the Hall sensor measurement decreases by $40 \%$ as the rotor is moved up $1 \mathrm{~mm}$ in the $y$ direction at the given sensor position.

The expected pattern of Hall sensor flux measurements can be predicted from Fig. 3(b) and the relative position between the sensor and PM under SE, DE, and ME conditions. If the rotor is concentric, the distance between the Hall sensor and all PMs are uniform as the rotor is rotated. The trajectory that shows the relative radial position between the Hall sensor and PM, as the rotor rotates, is shown in Fig. 4. Since the distance between the sensor and PMs is constant, the $\mathrm{N}$ pole (positive) and $\mathrm{S}$ pole (negative) peaks of the flux measurements are constant for a concentric rotor, as shown in the FE analysis results in Fig. 6(a).

Under SE conditions, the center of the rotor is offset from the center of the stator and the rotor rotates with respect to its own center. Therefore, the relative distance between the sensor and PMs is constant but different from that of the concentric case shown in Fig. 4. Fig. 5 shows the trajectory of the relative

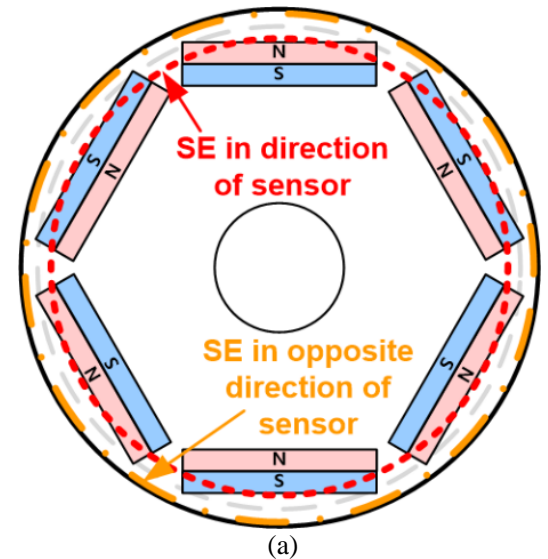

(a)

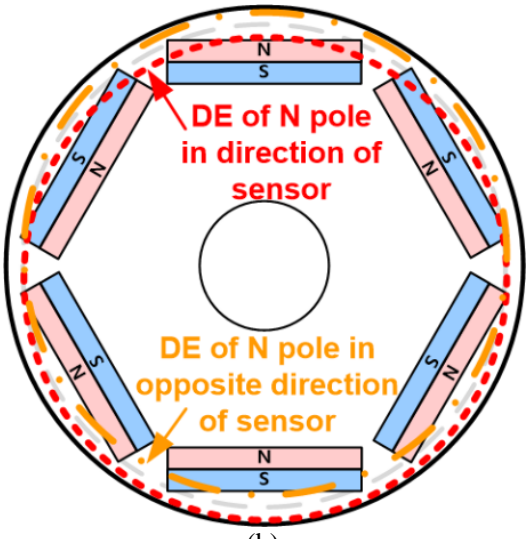

(b)

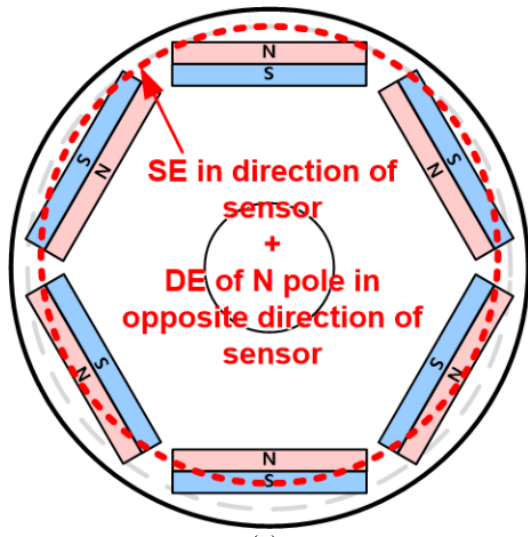

(c)

Fig. 5 Trajectories of the relative radial position between Hall sensor and PMs for motors with (a) SE, (b) DE, and (c) ME 
position between the sensor and PM for SE, DE and ME cases. The trajectory represents how the relative position between the PMs and sensor changes as the rotor is rotated. With SE, the size (diameter) of trajectory changes with the center identical to that of the concentric case, as shown in Fig. 5(a). The trajectory changes depending on the direction of rotor SE with respect to the Hall sensor. The trajectories for the two extreme cases of $\mathrm{SE}$, where the rotor is offset in the same and opposite directions ( $y$-axis direction in Fig. 2) of the sensor are shown in Fig. 5(a) in comparison to the concentric case. Since decrease in the radial distance between the sensor and PM (rotor moving up) results in a decrease in the measured flux, it can be predicted from Figs. 3 and 5(a) that the amplitude of the $\mathrm{N}$ and $\mathrm{S}$ pole peaks decrease uniformly, if SE is in the direction of Hall sensor (trajectory with decreased diameter in Fig. 5(a)). If SE is in the opposite direction of the Hall sensor, the $\mathrm{N}$ and $\mathrm{S}$ pole peaks increase uniformly (trajectory with increased diameter in Fig. 5(a)). The waveforms of the analog Hall sensor measurements obtained from the 3D FE with $30 \%$ and $60 \%$ SE in the direction of the Hall sensor are shown in Fig. 6(a).

Under DE conditions, the center of the rotor is offset from that of the stator, and the rotor rotates with respect to the center of the stator. The trajectory of the relative position between the sensor and PM depends on the direction of DE, as in the case of $\mathrm{SE}$. Since the rotor rotates with respect to the center of the stator, the center of the trajectory is shifted with its diameter identical to that of the concentric case. The trajectories under DE conditions with the DE of $\mathrm{N}$ pole in the same and opposite directions of the Hall sensor are shown in Fig. 5(b) along with the concentric case. From Figs. 3 and 5(b), it can be predicted that half $(p)$ of the adjacent peaks increase as the sensor is farther away from the PMs and the other half of the peaks decrease with $\mathrm{DE}$ as the sensor is closer to the PMs. This can be seen in the 3D FE analysis results of $30 \%$ and $60 \%$ DE with the $\mathrm{N}$ pole in the opposite direction of the sensor shown in Fig. 6(b). The first $3 \mathrm{~S}-\mathrm{N}-\mathrm{S}$ pole peaks increase in amplitude and next N-S-N pole peaks decrease in amplitude, as expected.

The flux pattern under ME conditions is more complicated as it is a combination of SE and DE. The size and center of the sensor-PM distance trajectory both change with ME. There are endless combinations of SE and DE that can produce ME. The case where $30 \% \mathrm{SE}$ is in the direction of the sensor and $30 \% \mathrm{DE}$ of the $\mathrm{N}$ pole is in the opposite direction of the sensor is illustrated as an example in Fig. 5(c) along with the trajectory of the concentric case. If the degree of SE and DE are identical, it can be predicted from Figs. 3 and 5(c) that the peak of the $\mathrm{N}$ pole flux on the top of Fig. 5(c) is identical to the concentric case, and the rest of the peaks decrease in amplitude since the Hall sensor is closer to the PM. The 3D FEA results of the flux measurements under this ME condition in Fig. 6(c) show that only one $\mathrm{N}$ pole peak in the flux is identical, and the other 5 peaks decrease with respect to the concentric case, as predicted. When compared to the case of 30\% SE, the first $3 \mathrm{~S}-\mathrm{N}-\mathrm{S}$ pole peaks increase and next N-S-N pole peaks decrease. The 3D FEA results show that the peaks of the $\mathrm{N}$ and $\mathrm{S}$ pole flux measurements are uniform for SE, but are non-uniform for DE and $\mathrm{ME}$ conditions due to the DE component present.

PM demagnetization has major influence on the Hall sensor measurements since it directly influences the flux. Uniform or symmetric demagnetization would result in uniform decrease in all the peaks in the measured PM flux similar to that of SE. On the other hand, local or asymmetric PM demagnetization is

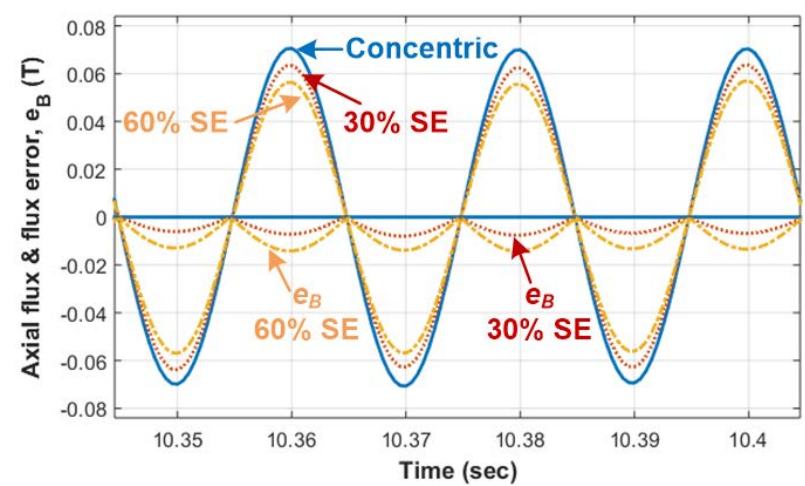

(a)

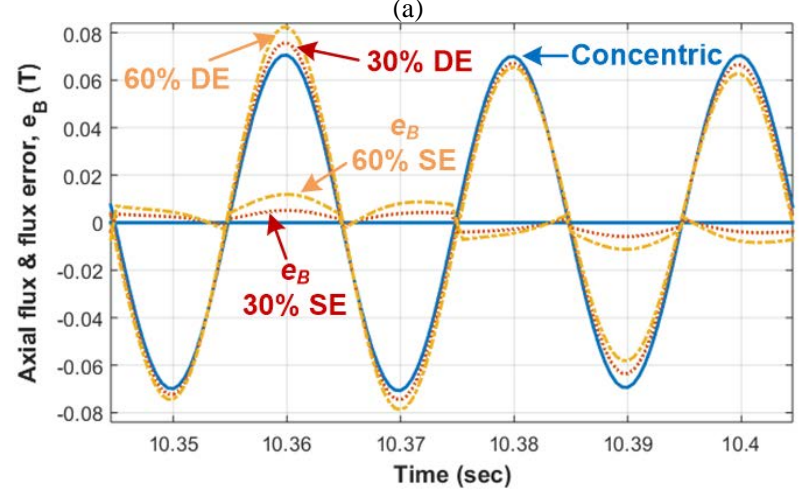

(b)

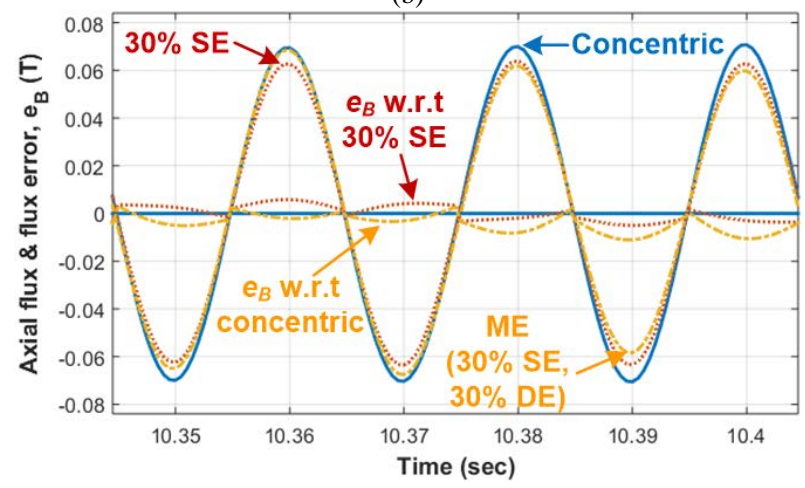

(c)

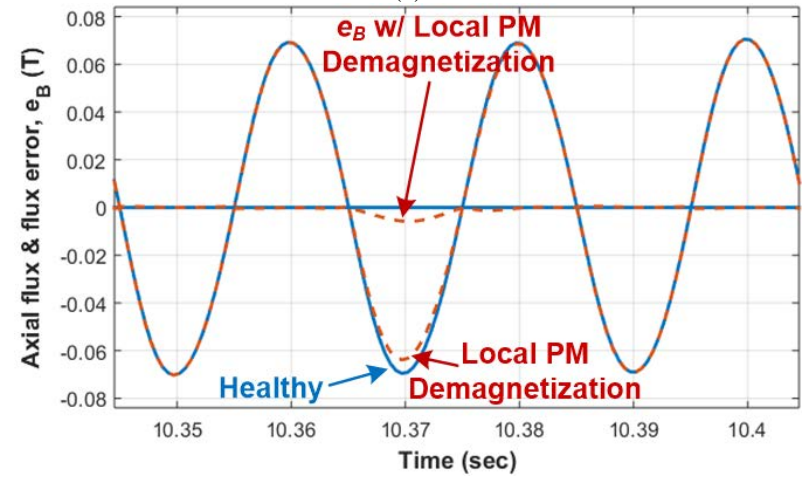

(d)

Fig. 6 3D FEA results of Hall sensor measurements and flux error, $e_{B}$, obtained for healthy rotor, and rotor with (a) $30 \%, 60 \%$ SE, (b) $30 \%$, $60 \% \mathrm{DE}$, (c) ME (30\% SE, 30\% DE), and (d) local demagnetization in one PM (5\%) 
expected to decrease the flux peaks of the corresponding PMs that have been demagnetized. The change in the measured flux pattern is therefore, easier to predict when local PM demagnetization occurs in the rotor. Local demagnetization condition was produced in the 3D FE model by reducing the magnetic strength of one PM by 5\%, and the FEA results are shown in Fig. 6(d). It can be clearly observed from this figure that the peak of the flux waveform measured from a healthy concentric rotor decreases when the pole with the demagnetized PM passes the Hall sensor. It can be seen that local PM demagnetization can be easily detected and distinguished from $\mathrm{DE}$ or ME conditions since only the peak(s) of the pole(s) with demagnetization is influenced.

\section{PRACTICAL CONSIDERATIONS}

Although it is shown in II that the Hall sensor measurement can be used for detecting rotor eccentricity and local demagnetization, there are some non-ideal issues to be considered for implementation. For reliable detection of eccentricity and demagnetization, it is important to understand how manufacturing defects/imperfections influence the proposed method. It is also important to consider the impact of PM temperature variation, stator endwinding current interference, measurement error, and temperature dependency of Hall sensor characteristics.

A manufacturing imperfection that could influence the proposed method is the mismatch in the magnetic strength or positioning of the PMs installed in the rotor. Although the flux peaks are well-balanced for most cases, the lower cost motors tend to have larger degree of asymmetry in the peaks even for a new motor with concentric rotor. To guarantee reliable fault detection in case the PMs are not symmetric, a fault detection algorithm based on monitoring the "change" in the flux peaks with respect to that obtained at commissioning is required.

There are other factors that influence the amplitude of the flux measurement such as PM temperature, stator endwinding current interference, temperature dependent Hall sensor characteristics, and sensor error/calibration issues [22]. The PM flux changes with PM temperature, and therefore, the Hall sensor measurement will also change with PM temperature. In addition, the Hall sensor output also depends on its temperature, although this can be reduced by using temperature compensated Hall sensors or by using constant current source [16], [22]. Furthermore, the sensor also picks up the leakage flux from the stator endwinding current in addition to the PM flux, and therefore, the measurement changes with the amplitude of the current (or load). These factors will degrade the sensitivity and reliability of the proposed method; therefore, the rotor fault detection algorithms must take these factors into account, as will be described in IV.

\section{Detection of DE, ME, AND LOCAl DEMAGNETiZATION}

\section{A. Reliable Rotor Fault Detection Algorithm}

Among the different types of eccentricity, SE is less critical to detect compared to $\mathrm{DE}$ and $\mathrm{ME}$, since its inherent level is low $(<10 \%)$, and does not increase in severity over time. However, it is desirable to detect $\mathrm{DE}$ and $\mathrm{ME}$ at incipient stages since the DE level can increase in severity, and is known to increase vibration and accelerate bearing wear and/or shaft bend that can lead to stator-rotor strike. The analysis of the flux measurements under eccentricity conditions in section II clearly shows that DE and ME can be detected from analog Hall sensor measurements. It is also important for the algorithm to account for nonuniformly magnetized PMs and the influence of temperature and load current variation for reliable diagnosis.

The flux density measurement, $B$, deviates from that obtained for the motor at commissioning, $B_{0}$, if $\mathrm{DE}$ is present. The difference, $e_{B}$, between $B$ and the stored values of $B_{0}$,

$$
e_{B}=|B|-\left|B_{0}\right|,
$$

can be monitored for detecting eccentricity as it deviates from zero as the DE level increases. The flux measurement is independent of speed, since the Hall sensor directly measures the amplitude of the flux unlike the flux coil. The FEA results of $e_{B}$ in the Hall sensor output for the concentric, SE, DE, and ME conditions are shown in Fig. 6. It is shown that the deviation in flux, $e_{B}$, increases with the severity of SE and DE. The pattern of $e_{B}$ for the case of ME where DE increases by 30\% from the $30 \%$ SE condition (Fig. 6(a)) is shown in Fig. 6(c). This represents the case where the inherent $\mathrm{SE}$ is $30 \%$ since SE usually does not increase with time $\left(B_{0}\right.$ is the flux measurement under 30\% SE). The pattern of $e_{B}$ in Fig. 6(c) is similar to that of the case where DE increases by $30 \%$ from the concentric condition shown in Fig. 6(b) since they both represent a $30 \%$ increase in DE.

Monitoring the "change" in the flux measurements from $B_{0}$ allows sensitive detection of eccentricity for cases where the flux measured from the multiple PMs of a concentric and healthy rotor are not symmetrical. However, this simple method is not immune to variation in $B_{0}$ with temperature and current interference, as described in III. A simple means of compensating for the temperature variation and current interference is to normalize the flux measurements with respect to itself. The rms values of the positive ( $\mathrm{N}$ pole) and negative (S pole) half cycles of the flux measurements can be obtained as

$$
\boldsymbol{m}=\left[M_{S 1} M_{N 1} M_{S 2} M_{N 2} M_{S 3} M_{N 3}\right]
$$

for the 6 pole motor measurements shown in Fig. 6. $M$ represents the positive and negative half cycle rms values, subscripts $S$ and $N$ represent poles of the magnet, and subscripts $1,2,3, \ldots$ are the pole pair numbers. Matrix $\boldsymbol{m}$ can be normalized with respect to the average value of its elements, $M_{a v g}$, as

$$
\boldsymbol{m}_{\boldsymbol{n}}=\left[M_{S 1} M_{N 1} M_{S 2} M_{N 2} M_{S 3} M_{N 3}\right] / M_{\text {avg }} .
$$

The normalized half-cycle rms values, $\boldsymbol{m}_{\boldsymbol{n}}$, are expected to be insensitive to variation in PM temperature, endwinding current interference, Hall sensor gain variation and sensor error since the elements of $\boldsymbol{m}$ change uniformly with the external factors. The variation in PM temperature due to increase in motor operating temperature can be assumed to be uniform for all PMs, and that it influences the values of the $\boldsymbol{m}_{\boldsymbol{n}}$ elements in (5) by the same ratio. Change in the Hall sensor gain also influences all positive and negative half cycle waveforms in the same way. The endwinding current also increases or decreases the PM flux measurement by a similar amount depending on the load [22]. Therefore, $\boldsymbol{m}_{\boldsymbol{n}}$ calculated from $\boldsymbol{B}_{0}, \boldsymbol{m}_{\boldsymbol{n} \mathbf{0}}$, is ideally constant, and is not influenced by the external non-ideal factors. 
Since it is the "change" in $\boldsymbol{m}_{\boldsymbol{n}}$ that is being monitored for anomalies in the flux, the difference between the presently measured and calculated $\boldsymbol{m}_{\boldsymbol{n}}$ and the stored $\boldsymbol{m}_{\boldsymbol{n} \boldsymbol{0}}$ of a new motor, shown in (6), can provide a reliable indication of DE, ME, and local demagnetization.

$$
\boldsymbol{e}_{\boldsymbol{m} \boldsymbol{n}}=\boldsymbol{m}_{\boldsymbol{n}}-\boldsymbol{m}_{\boldsymbol{n} 0} .
$$

The elements of $\boldsymbol{e}_{\boldsymbol{m} \boldsymbol{n}}$ will consist of small terms close to zero, if the $\mathrm{DE}$ level does not increase. If the $\mathrm{DE}$ level increases, the pattern of $\boldsymbol{e}_{\boldsymbol{m}}$ resembles the $\boldsymbol{e}_{B}$ pattern for the cases of DE and ME (Figs. 6(b)-(c)), where half of the peaks increase and half of the peaks decrease, as shown in Fig. 7. The pattern of $\boldsymbol{e}_{\boldsymbol{m} n}$ for local demagnetization also resembles the $e_{B}$ pattern where only the peak(s) that correspond to the demagnetized PM(s) decreases. An example of the $\boldsymbol{e}_{\boldsymbol{m} n}$ pattern for the example of one demagnetized PM is shown in Fig. 13(b) of section V.B. The advantage of using the $\boldsymbol{e}_{\boldsymbol{m} n}$, which is calculated by normalizing the data and taking the difference, is not clear for the ideal motor assumed in the FEA. However, its immunity to asymmetric PMs and interference due to temperature and current will be shown in the experimental results.

\section{B. Alternative Option for Digital Implementation}

Although analog signals can provide a clear indication of DE, $\mathrm{ME}$, and local demagnetization, the requirement of an analog Hall sensor and analog measurement may be undesirable for low-cost PMSM drive systems. An alternative option is to add analog circuitry to process the analog Hall sensor signal prior to digitization in a way such that the digitized signal contains information regarding DE, ME, and local demagnetization. One conceivable way of accomplishing this is to compare the rectified analog Hall sensor measurement with a predetermined offset signal before digitization, as shown in Fig. 8(a). If the rotor is concentric and ideal, the width of the pulses generated would be identical for each half cycle, as shown in Fig. 8(b)-(d). If the peaks produced by the PMs increase or decrease due to rotor eccentricity, the width of the digital pulses will increase or decrease for a given offset. The digitized signal of the comparator output can be acquired using a capture type digital input. The change in the widths of the pulses can be analyzed to detect anomalies produced by DE or local demagnetization. This is required for only one of the three phases.

Examples of the change in the digitized comparator output under 30\% DE with respect to the concentric case, and change under ME (30\% SE and 30\% DE) with respect to 30\% SE conditions are shown in Figs. 8(b)-(c), respectively. It can be seen in Figs. 8(b)-(c) that the width of half of pulses increase and half of the pulses decrease since the peaks of the measured flux increase or decrease in the same manner (Figs. 6(b)-(c)). For

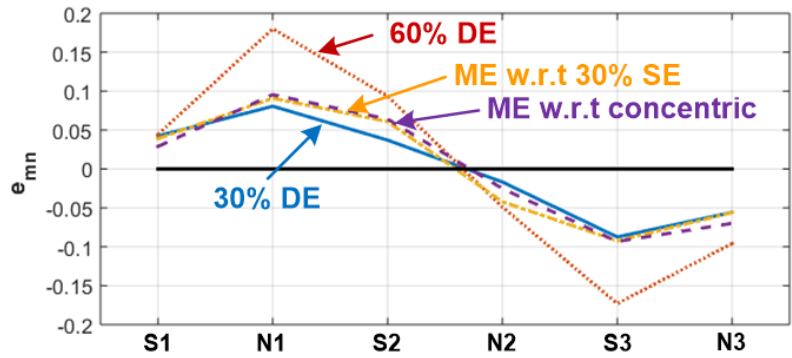

Fig. 7 Values of $\boldsymbol{e}_{m n}$ elements for $30 \%$ DE, 60\% DE, and ME conditions local demagnetization, the digitized pulse output that corresponds to the PM demagnetized by $5 \%$ disappears, as shown in Fig. 8(d). This condition can be easily detected since there will be a noticeable decrease in the width of the pulse that

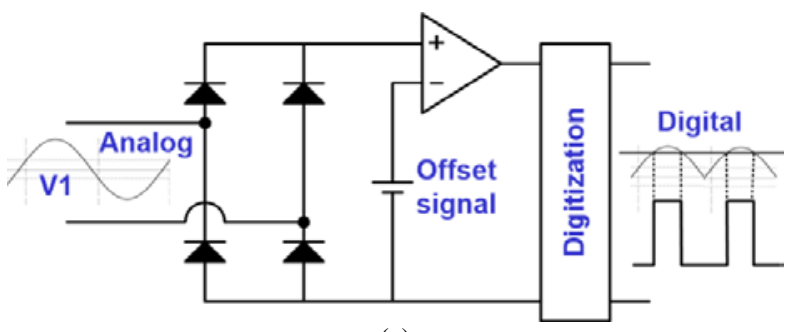

(a)
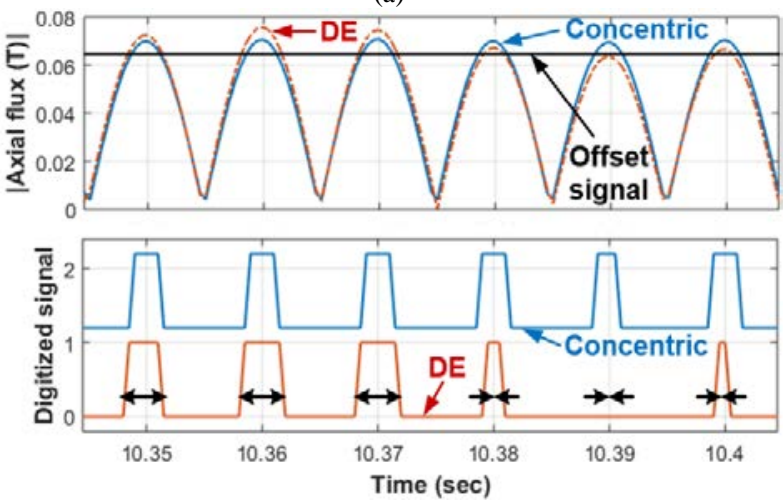

(b)
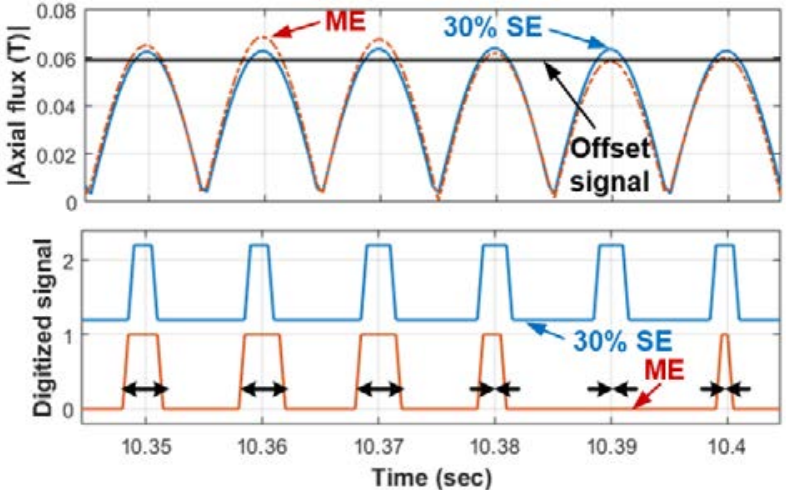

(c)
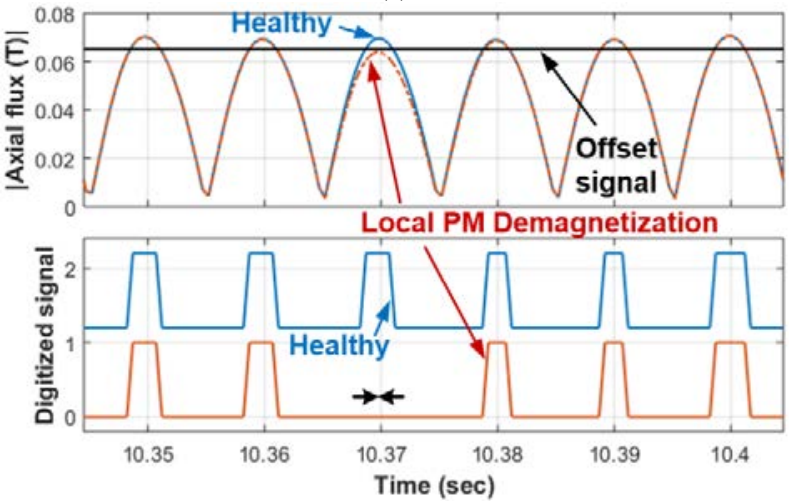

(d)

Fig. 8 (a) Schematic of alternative option for low cost implementation of proposed method with digital input; change in the digitized comparator output under (b) 30\% DE with respect to concentric, (c) ME (30\% SE and $30 \% \mathrm{DE}$ ) with respect to $30 \%$ SE conditions, and (d) local PM demagnetization conditions ( $5 \%$ demagnetization in one PM) 
corresponds to demagnetized PM, whereas the widths of the other pulses do not change.

Fig. 8 shows that the requirements for additional hardware can be simplified compared to when analog signal is available. However, there is not much flexibility in compensating for the influence of asymmetric PMs, or variation in temperature and current as in the case of the analog Hall sensor measurement. If the PMs are not symmetrical, all peaks of the rectified voltage and the width of all digital pulses shown in Figs. 8(b)-(d) are different. Since the variation in PM/sensor temperature or load influence all the peaks uniformly, the widths of the pulses either increase or decrease simultaneously with the non-ideal external factors. The digital input signals can be processed to compare the "change" in the width of the pulses under the same load condition to detect DE or local demagnetization. Digital input based detection of DE with asymmetric PMs under the same load condition is demonstrated in V.C.

\section{EXPERIMENTAL RESULTS}

\section{A. Experimental Test Setup}

Experimental verification of the proposed method was performed on a $2.2 \mathrm{~kW}, 212 \mathrm{~V}, 4500 \mathrm{rpm}, 6$ pole IPMSM. The end shields of the test machine were replaced with custom designed and built shields to emulate artificial fault conditions, and to install an analog Hall sensor and encoder, as shown in Fig. 9. To produce eccentricity conditions, inner and outer bearing sleeves were installed on the bearings to fit between the rotor shaft and end shield, as shown in Fig. 9. The inner hole of the inner and outer sleeves were precision machined and offset from the center by $0 \%$ and $30 \%$ of the $0.7 \mathrm{~mm}$ airgap to produce SE and DE conditions, respectively, on both sides of the motor. ME condition was produced with a combination of SE and DE sleeves. To produce local demagnetization conditions, 3\% of one PM was chipped off as shown in Fig. 9(c). Flux in the axial direction was measured with the Hall sensor attached to a rod protruding inward from the end shield, as shown in Fig. 9. The rod was designed so that the sensor is positioned $3.2 \mathrm{~mm}$ apart from the PM in the axial direction and $3.5 \mathrm{~mm}$ above the center of the PM in the radial direction. A commercial Hall sensor with resolution of $3.125 \mathrm{~V} / \mathrm{G}$ was installed at the end of the rod as shown in 9. A 320F28335 $\mu$-controller was used for controlling the IGBT inverter and acquisition of the Hall sensor and current data at $6.4 \mathrm{kHz}$. The motor was operated at no load, half load, and rated load under all fault conditions.

\section{B. Experimental Results - Analog Measurement}

The Hall sensor analog measurements for the concentric and healthy rotor under no load, half load, and full load conditions are shown in Fig. 10(a) to show how the flux is influenced by endwinding current (and PM temperature). It can be seen that the flux for the 6 half cycles increase or decrease in a similar manner with load. The elements of $\boldsymbol{m}_{\boldsymbol{n}}$, which are the normalized half cycle rms values of the flux calculated from (5) are shown in Fig. 10(b). The $\boldsymbol{m}_{\boldsymbol{n}}$ elements are not influenced by the endwinding load current since they are normalized. Since it is the measurement for a concentric motor, it can serve as the reference matrix, $\boldsymbol{m}_{\boldsymbol{n} \mathbf{0}}$, for obtaining the fault indicator $\boldsymbol{e}_{\boldsymbol{m} \boldsymbol{n}}$ as described in IV.A. The average of the $\boldsymbol{m}_{\boldsymbol{n}}$ measurements under the 3 load conditions was used as $\boldsymbol{m}_{\boldsymbol{n} \mathbf{n}}$.
The flux measurements for the motor with concentric and $30 \%$ DE rotors under full load conditions are shown in Fig. 11(a). $\mathrm{DE}$ of the $\mathrm{N}$ pole was set in the opposite direction of the Hall sensor to make it identical to the FE results presented in II. The results show that there are differences in the $\mathrm{N}$ and $\mathrm{S}$ pole peaks of the flux even for the concentric case due to inherent asymmetry in PMs; however, the reliability of the method is not influenced if the "change" in the peaks is monitored. The peaks

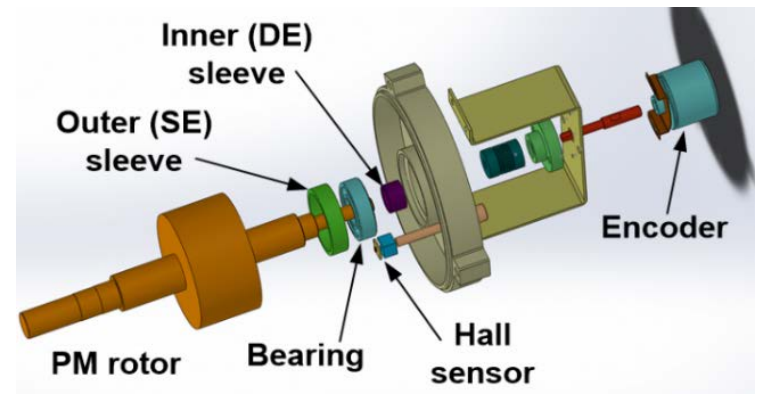

(a)

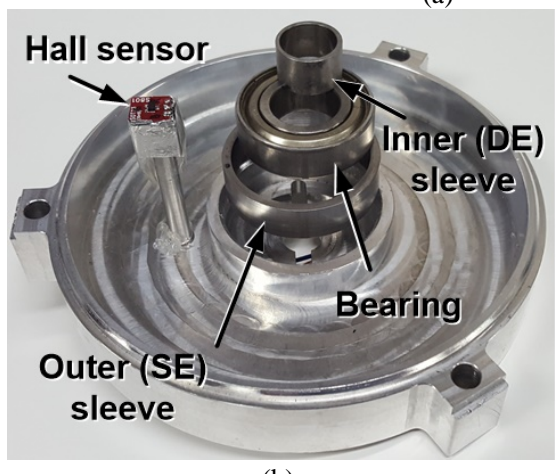

(b)

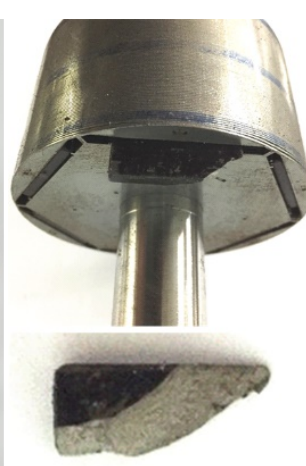

(c)
Fig. 9 Experimental setup: (a) design drawing; (b) custom designed and built end shield for introducing SE, DE, and ME conditions, Hall sensor, and encoder; and (c) chipped PM for emulating local demagnetization

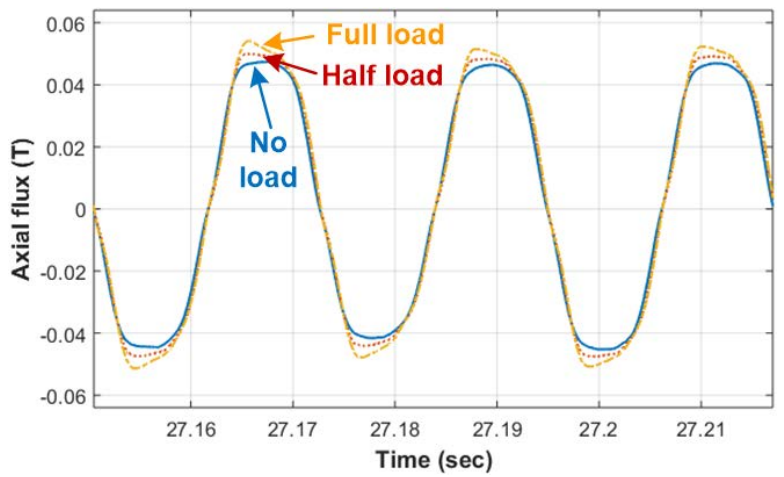

(a)

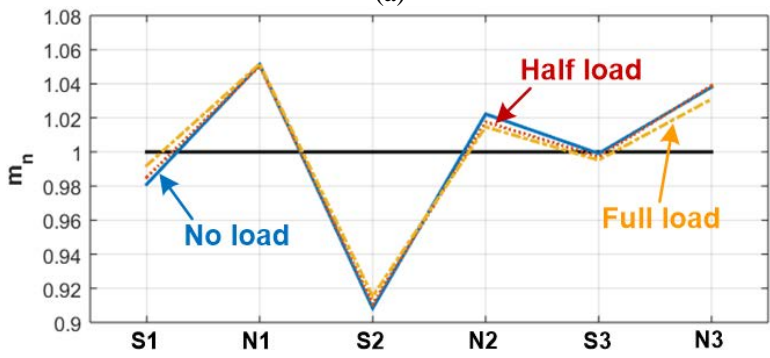

(b)

Fig. $10 \quad$ (a) Hall sensor analog flux measurements and (b) elements of $m_{n}$ for the concentric rotor under no load, half load, and full load conditions 
of the $\mathrm{N}$ and $\mathrm{S}$ poles change as predicted in Figs. 6(b) under 30\% $\mathrm{DE}$, in that the amplitude of the first three 3 peaks increase, and the amplitudes decrease for the next 3 peaks. The fault indicator $\boldsymbol{e}_{\boldsymbol{m} n}$ was calculated from $\boldsymbol{m}_{\boldsymbol{n} \boldsymbol{0}}$ and the $\boldsymbol{m}_{\boldsymbol{n}}$ matrices obtained from the concentric and 30\% DE cases under no load, half load, and full load conditions, as shown in Fig. 11(b). It should be noted that the information on the reference $\mathrm{N}$ pole is required for comparison when monitoring the change, if the peaks are not identical. This can be done by comparing the present $m_{n}$ to the

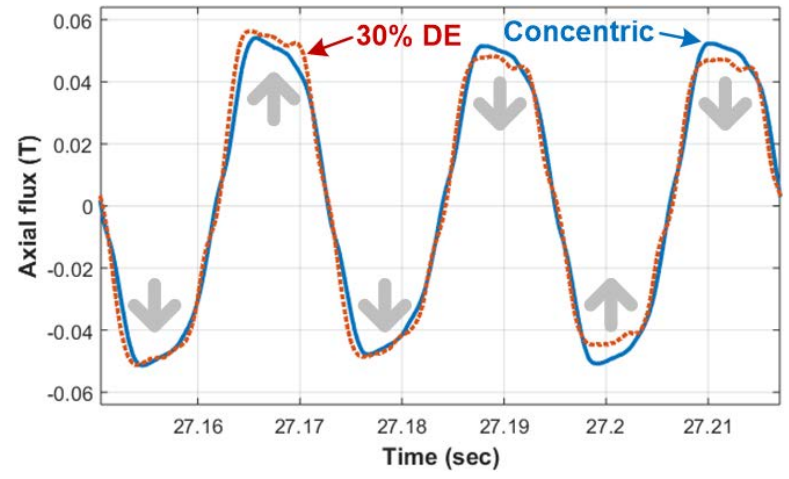

(a)

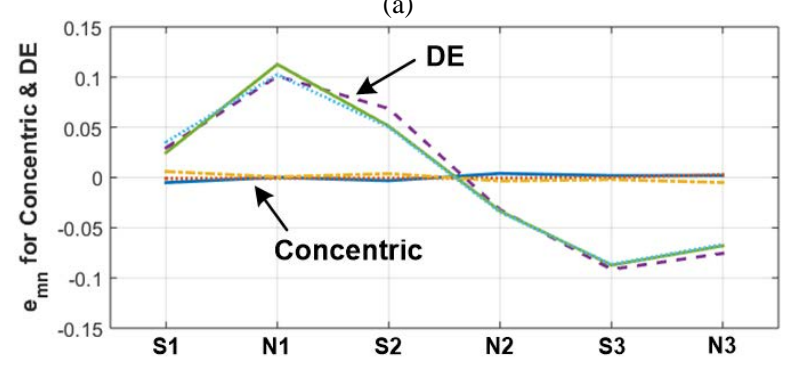

(b)

Fig. 11 (a) Flux measurements (full load); (b) elements of $e_{m n}$ (no load, half load, full load) under concentric and DE conditions

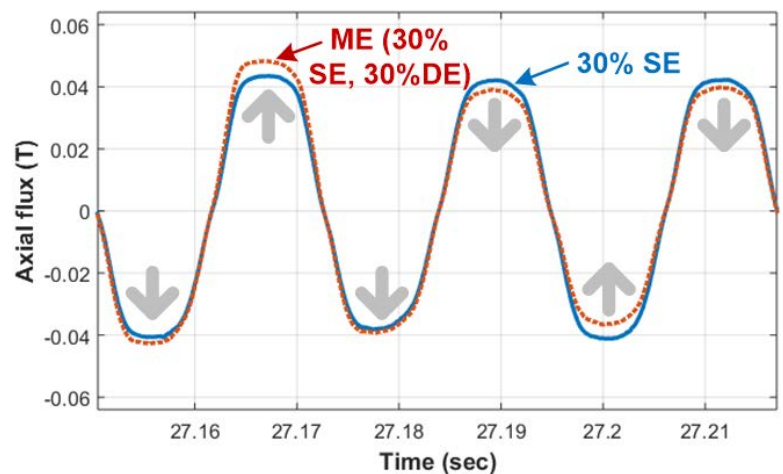

(a)

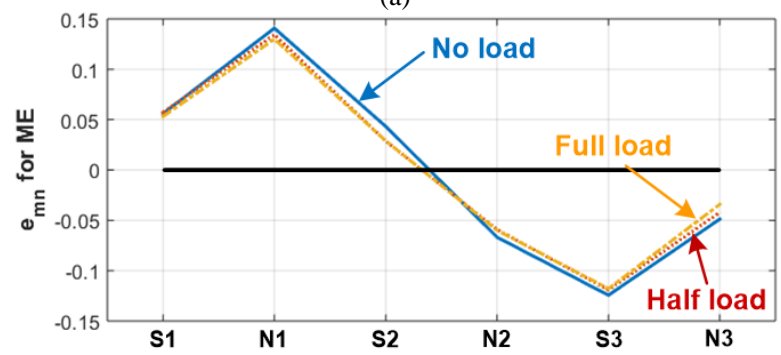

(b)

Fig. 12 (a) Flux measurements (no load); (b) elements of $e_{m n}$ (no load, half load, full load) under $\mathrm{ME}$ (30\% SE, 30\% DE) with respect to $30 \% \mathrm{SE}$ previous $m_{n}$, which will be similar, since the DE level or demagnetization progresses slowly with time. The results clearly show that $\boldsymbol{e}_{\boldsymbol{m}}$ is close to zero for the concentric rotor and produce a sinusoidal pattern similar to the FE results in Fig. 7 for DE regardless of the load condition. This confirms that the proposed fault indicator provides indication of $\mathrm{DE}$ and is immune to load and temperature variations.

The flux measurements under ME conditions (with 30\% SE in the direction of the sensor and $30 \% \mathrm{DE}$ of the $\mathrm{N}$ pole in the opposite direction of the sensor) are shown in Fig. 12(a) when the motor is operating under no load conditions. The change in the $\mathrm{N}$ and $\mathrm{S}$ pole peaks with respect to the $30 \% \mathrm{SE}$ condition are identical to the predictions made from Fig. 6(c). The values of $\boldsymbol{e}_{\boldsymbol{m} n}$ shown in Fig. 12(b) are also consistent with the FE based predictions shown in Fig. 7, i.e. it produces a similar sinusoidal pattern for ME under all load conditions. The trend in the it is expected to change in $\mathbf{e}_{\boldsymbol{m} n}$ with $30 \%$ increase in DE is identical whether it increase from that of a concentric motor or from a motor with $30 \% \mathrm{SE}$, regardless of the load condition. Although the results for $30 \%$ DE level were shown, it can be deduced from Fig. 3(b) that the fault indicator would be proportional to the severity of DE. Since the peaks of $e_{m n}$ are proportional to $\mathrm{DE}$ level and $e_{m n}$ has a unique pattern, the proposed method is expected to provide sensitive and reliable detection of $\mathrm{DE}$.

The Hall sensor measurement over one mechanical revolution of the rotor with the one of the magnets (S2) chipped (Fig. 9(c)) is shown in Fig. 13(a) under full load operating conditions. It can be seen that the flux measurement decreases only for the pole under which the PM is demagnetized, as predicted. There is a significant decrease in one of the values of $\boldsymbol{e}_{\boldsymbol{m} n}$ that corresponds to the S2 demagnetized PM, as shown in Fig. 13(b), under no load, half load, and full load conditions. The nonzero increase in the $\boldsymbol{e}_{\boldsymbol{m} n}$ elements not related to S2 is caused by the normalization of $m_{n}$ in (5) with a decreased value

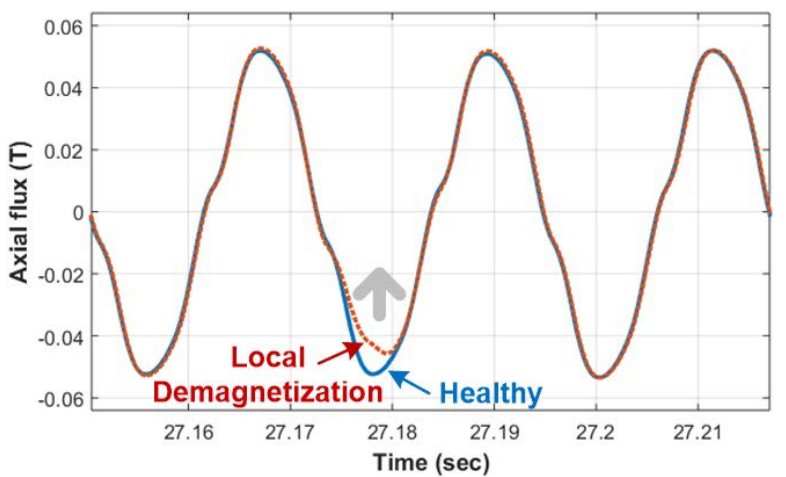

(a)

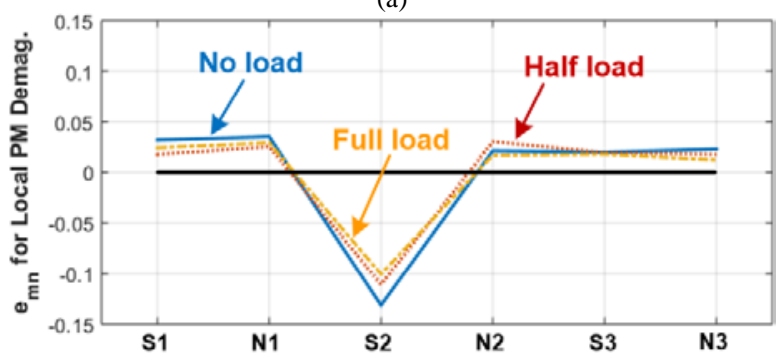

(b)

Fig. 13 (a) Flux measurements (full load); (b) elements of $e_{m n}$ (no load, half load, full load) under local demagnetization in S2 PM 


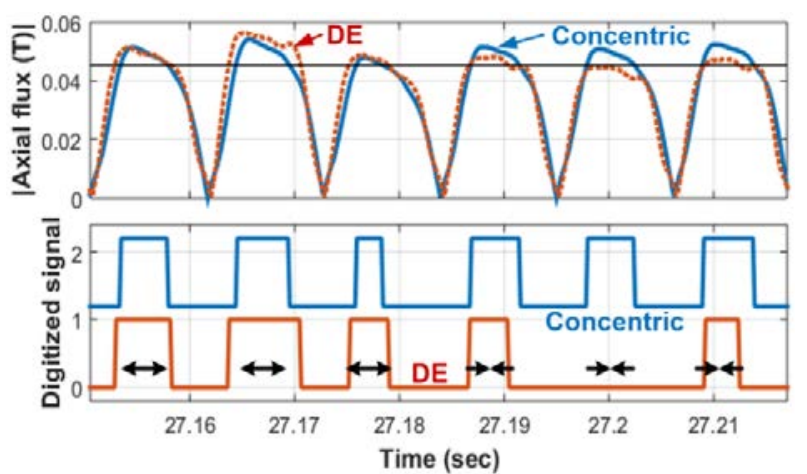

Fig. 14 Change in the digitized comparator output under $30 \%$ DE with respect to concentric condition under full load condition
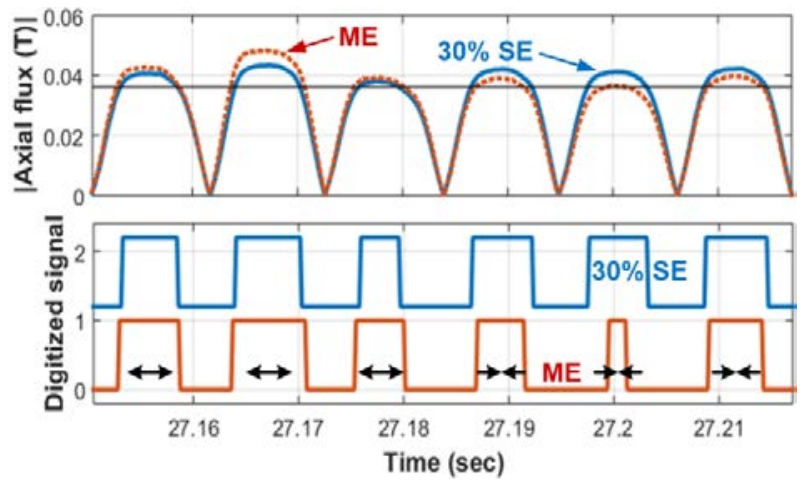

Fig. 15 Change in the digitized comparator output under ME (30\% SE and $30 \% \mathrm{DE}$ ) with respect to $30 \%$ SE conditions under no load condition

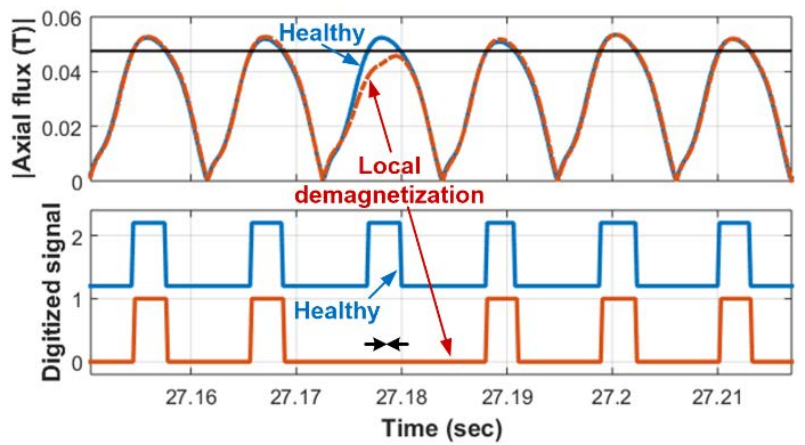

Fig. 16 Change in the digitized comparator output under local PM demagnetization in one magnet under full load condition

of $M_{\text {avg. }}$. Nevertheless, the noticeable decrease in one element is sufficient for detecting local demagnetization independent of the load condition.

\section{Experimental Results - Digital Measurement}

The detectability of DE, ME, and local demagnetization with the alternative option of using digitized signals was also verified by post-processing the analog signals. The change in the digitized comparator output for the same DE, ME, and local demagnetization cases as the analog signals (Figs. 11,12, and 13) are shown in Figs. 14, 15, and 16, respectively. Since the peaks of the analog signals are not uniform, the width of the pulses for a constant offset signal are not uniform. However, the pattern of change in the pulse widths with respect to that of the reference pulse widths at the same load conditions is consistent. For both cases of 30\% DE with respect to the concentric rotor and $\mathrm{ME}$ with respect to the $30 \% \mathrm{SE}$ rotor, the width of the first 3 pulses increase and the next 3 pulses decrease. Fig . 16 shows that the pulse under the demagnetized PM disappears since its amplitude decreases below the predetermined offset making detection very clear. Change in other factors under a given load condition would cause an increase or decrease in the width of all pulses.

The offset signal for digital implementation can be set at a value close to the peak value. According to the authors' experience, it was possible to detect all the rotor faults tested when the offset was set between $85 \%$ to $90 \%$ of the average of the peaks. The knowledge on the reference position of the $\mathrm{N}$ or $S$ pole is required for the monitoring the change in the pulsewidth. An algorithm that compares the widths of present and previous pulses can be devised. The results are identical to the FE results shown in Figs. 8(b)-(c), respectively, which shows that the proposed method can provide reliable detection of increase in DE level and local demagnetization.

\section{CONCLUSION}

A new method for detecting rotor eccentricity and local demagnetization for PMSMs based on the analog Hall sensor signals available in the motor, was presented in this paper. Verification with 3D FEA and experimental testing of an IPMSM under controlled DE, ME, and local demagnetization conditions showed that the proposed method can provide sensitive detection of rotor faults, since it is based on direct measurement of PM flux inside the motor. An alternative option for low cost implementation based on digital input signal has also been provided. The practical issues for implementation have also been identified, resolved, and verified for reliable detection in the field. The results are meaningful considering that existing on-line methods based on spectrum analysis of current or vibration cannot detect rotor faults for all PMSM designs. The proposed method is expected to provide reliable detection since it does not require motor model information, and is immune to the influence of motor design, ambient or operating conditions.

\section{REFERENCES}

[1] T. Goktas, M. Zafarani, K.W. Lee, B. Akin, T. Sculley, “Comprehensive analysis of magnet defect fault monitoring through leakage flux," IEEE Trans. on Magn., vol. 53, no. 4, pp. 1-10, April 2017.

[2] M. Delgado Prieto, et. al., "Feature extraction of demagnetization faults in PMSMs based on box-counting fractal dimension,” IEEE Trans. on Ind. Electr., vol. 58, no. 5, pp. 1594-1605, May 2011.

[3] W. le Roux, R.G. Harley, T.G. Habetler, "Detecting rotor faults in low power permanent magnet synchronous machines," IEEE Trans. Power Electr., vol. 22, no. 1, pp. 322-328, Jan. 2007.

[4] S. Rajagopalan, W. le Roux, T. G. Habetler, R. G. Harley, "Dynamic eccentricity and demagnetized rotor magnet detection in trapezoidal flux (brushless DC) motors operating under different load conditions," IEEE Trans. Power Electr., vol. 22, no. 5, pp. 2061-2069, Sept. 2007.

[5] B.M. Ebrahimi, J. Faiz, "Magnetic field and vibration monitoring in permanent magnet synchronous motors under eccentricity fault," IET Elec. Power Appl., vol. 6, no. 1, pp. 35-45, January 2012.

[6] W. Doorsamy, A.A.E. Abdallh, W.A. Cronje, L. Dupré, “An experimental design for static eccentricity detection in synchronous machines using a Cramér-Rao lower bound technique," IEEE Trans. on Ener. Conv., vol. 30, no. 1, pp. 254-261, March 2015.

[7] B.M. Ebrahimi, J. Faiz, B.N. Araabi, "Pattern identification for eccentricity fault diagnosis in permanent magnet synchronous motors using stator current monitoring," IET Electr, Power Appl., vol. 4, no. 6, pp. 418-430, July 2010. 
[8] T. Goktas, M. Zafarani, B. Akin, "Discernment of broken magnet and static eccentricity faults in permanent magnet synchronous motors," IEEE Trans. Energy Conv., vol. 31, no. 2, pp. 578-587, June 2016.

[9] J. Rosero, et. al, "Fault detection of eccentricity and bearing damage by means of wavelet transforms decomposition of the stator current" Proc. of IEEE APEC, pp. 111-116, 2008.

[10] S. Rajagopalan, et. al., "Nonstationary motor fault detection using recent quadratic time-frequency representations," IEEE Trans. on Ind. Appl., vol. 44, no. 3, pp. 735-744, May/June 2008.

[11] J. Hong, et. al., "Detection and classification of rotor demagnetization and eccentricity faults for PM synchronous motors,” IEEE Trans. Ind. Appl., vol. 48, no. 3, pp. 923-932, May/June 2012.

[12] Y. Park et al., "On-line detection of rotor eccentricity for PMSMs based on hall-effect field sensor measurements," Proc. of IEEE ECCE, pp. 4678-4685, 2017.

[13] J.R. Cameron, W.T. Thomson, A.B. Dow, "Vibration and current monitoring for detecting air gap eccentricity in large induction motors," Proc. Inst. Elect. Eng., vol. 133, no. 3, pt. B, pp. 155-163, May 1986.

[14] Faulhaber.com, “Brushless Dc servomotors,” Series 22 BX4323268BX4, Mar. 2017. [Online]. Available: https://fmcc.faulhaber.com

[15] Technosoft.com, "Drive solutions for motion control applications product overview,”, Mar. 2017. [Online]. Available: http://www.technosoftmotion.com/en/products
[16] Allegro micro systems LLC, "Low noise, linear hall effect sensor ICs with analog output,” A1324, A1325, and A1326, Mar. 23, 2017. [Online]. Available: http://www.allegromicro.com

[17] Y. F. Shi, Z. Q. Zhu, D. Howe, "EKF-based hybrid controller for PM brushless motors combining hall sensors and a flux-observer-based sensorless technique,” Proc. of IEEE-IEMDC, pp. 1466-1472, May 2005.

[18] A. Simpkins, E. Todorov, "Position estimation and control of compact BLDC motors based on analog linear hall effect sensors," Proc. of American Control Conference, pp. 1948-1955, July 2010.

[19] X. Song, J. Fang, B. Han, "High-precision rotor position detection for high-speed surface PMSM drive based on linear hall-effect sensors," IEEE Trans. Power Electron., vol. 31, no. 7, pp. 4720 -4731, July 2016.

[20] Y. Y. Lee, R.-H. Wu, S.T. Xu, “Applications of linear hall-effect sensors on angular measurement," Proc. of IEEE Int'l Conf. on Control Appl. (CCA), pp. $479-482$, Sept. 2011.

[21] L. Xiao, Y. Yunyue, Z. Zhuo, "Study of the linear hall-effect sensors mounting position for PMLSM,” Proc. of IEEE Conf. on Ind. Electr. and Appl., pp. 1175 - 1178, May 2017.

[22] D. Fernandez, et. al., "Permanent magnet temperature estimation in PM synchronous motors using low cost hall effect sensors," IEEE Trans. on Ind. Appl., vol. 53, no. 5, pp. 4525-4524, Sept./Oct. 2017. 